\title{
A case of adult-onset leukoencephalopathy with axonal spheroids and pigmented glia (ALSP) with a high antinuclear antibody titer
}

\author{
Shitan Zheng ${ }^{1} \mathbb{D} \cdot$ Juanjuan Wang ${ }^{2}$
}

Received: 18 February 2021 / Accepted: 22 September 2021 / Published online: 27 September 2021

(c) Fondazione Società Italiana di Neurologia 2021

\section{Dear Editor,}

Adult-onset leukoencephalopathy with axonal spheroids and pigmented glia (ALSP) is an autosomal dominant white matter disease caused by mutations in the colony-stimulating factor 1 receptor gene (CSF1R). It is characterized clinically by adult-onset behavioral, cognitive, and motor dysfunction. Neuropsychiatric (NP) involvement is one of the most complex and challenging features of SLE because none of the NP syndromes that occur in SLE have specific features. Here, we report a patient with ALSP carrying a high titer of antinuclear antibody who was initially misdiagnosed as NP-SLE.

The patient was a 47-year-old woman who was referred to our university hospital because of progressive left limb weakness and cognitive impairment for 2 months. Memory decline and decreased calculation ability were also noticed. On neurological examination, the muscle strength of the left limb was grade 4, with a left positive Babinski sign. Frontal gait disorder and involuntary tremor of the left upper limb and right lower limb were observed. Notably, her mother and younger brother had similar clinical symptoms of hemiparalysis and died in middle age.

Past medical history showed an SLE diagnosis 1 year earlier at another clinic because of her dizziness, thrombocytopenia, elevated titer of $\beta 2$-GPI $(57.81 \mathrm{RU} / \mathrm{ml})$ and high titer of ANA (1:1000), and U1RNP $(+++)$. Brain MRI at that time showed multiple infarctions in bilateral frontoparietal areas, but we did not obtain images. She had received high-dose glucocorticoid and cyclophosphamide therapy,

Shitan Zheng

janeone@163.com

1 Department of Emergency, The Second Affiliated Hospital of Fujian Medical University, No. 950 Donghai Street, Quanzhou, Fujian Province, China

2 Department of Rheumatology, The Second Affiliated Hospital of Fujian Medical University, Quanzhou, China but she did not visit the hospital for half a year because of the COVID-19 epidemic and stopped taking all of the drugs.

Serum ANA at this time still showed a high titer of 1:1000, and ENA showed U1RNP +++ . The platelet count was $97 * 10^{\wedge} 9 / \mathrm{L}$, however, this was determined to be pseudothrombocytopenia when the anticoagulant was changed from EDTA to sodium citrate. ACL and $\beta 2$-GPI were negative. C3, C4, ESR, and CRP were normal. Brain MRI showed bilateral white matter lesions in periventricular areas and the corpus callosum (Fig. 1C and D). No enhanced lesions were seen with the enhancement sequence. MRA and MRV did not show obvious abnormalities. Routine and biochemical results of cerebrospinal fluid were normal.

First, she was diagnosed with NP-SLE, and pulse therapy with glucocorticoids and cyclophosphamide was used again, with low-molecular-weight heparin for APS was suspected to accompany the diagnosis. However, her motor and cognitive symptoms deteriorated, and she started to have fecal and urinary incontinence. Then, we contacted the clinic where her first brain MRI was examined, and we finally obtained her MRI image (Fig. 1A and B). The neuroimage from 1 year before had already shown multiple, patchy, bilateral, and hyperintense lesions in periventricular areas, the corpus callosum, and deep white matter regions of the frontal and parietal lobes. By the present time, the abnormal signals became slightly more widespread and confluent.

Next-generation sequencing was performed because of her family history, diffuse white matter lesions, poor treatment effect, and deterioration of disease. The results revealed a novel heterozygous variation, c.2467G > A (p.A823T), in the CSF1R gene (Fig. 2). Therefore, a definitive diagnosis of ALSP was made. The neurological manifestations were finally attributed to ALSP. So the diagnosis of SLE was not fulfilled, and all of the drugs for SLE were downregulated.

ALSP is a progressive degenerative white matter disorder. The mean age at onset of ALSP was reported to be $43 \pm 11$ years [1]. It is a rare disease and has various clinical presentations, such as parkinsonism, pyramidal signs, 


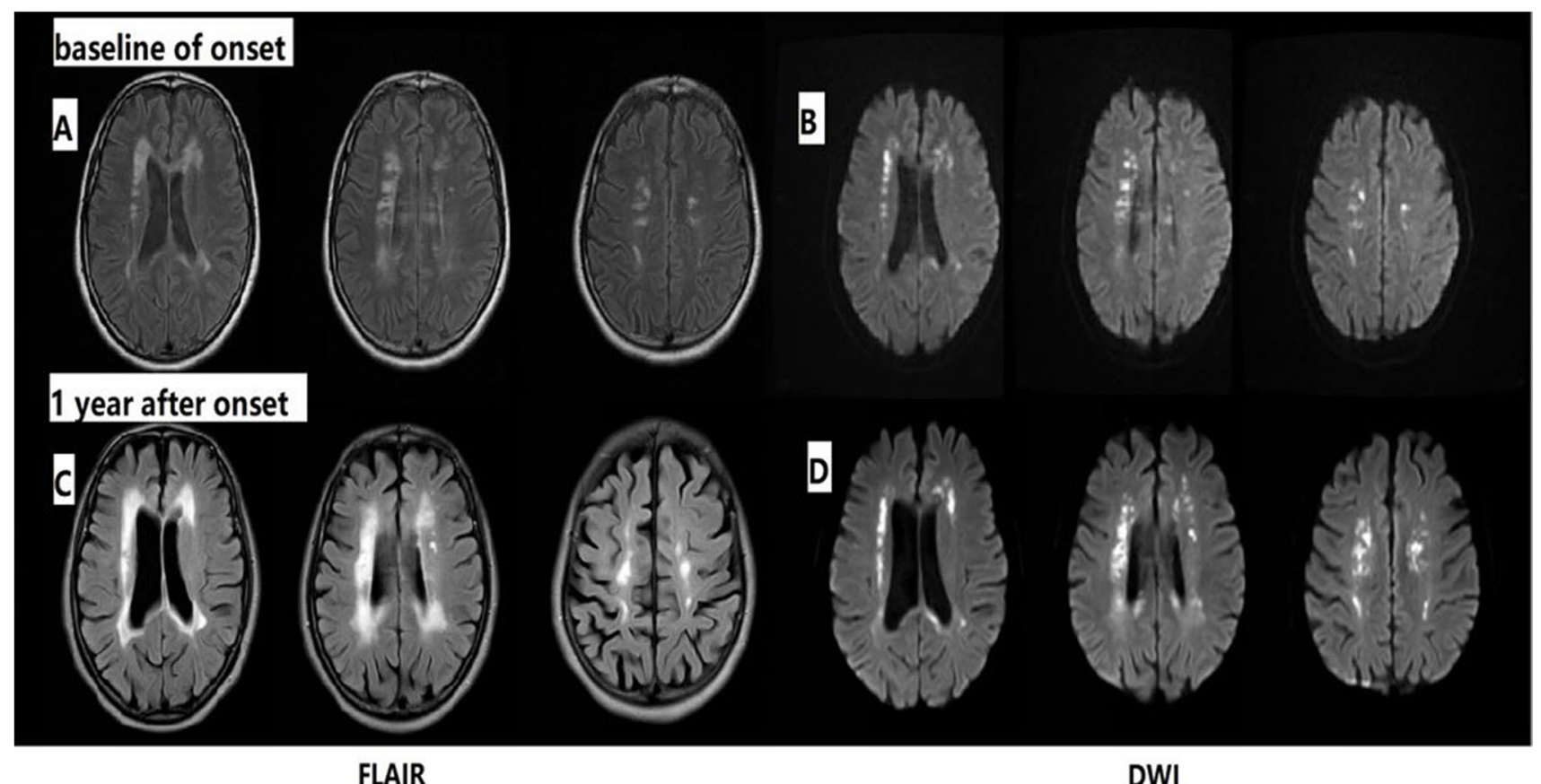

Fig. 1 MRI of the brain. A, B Brain FLAIR and DWI MRI showed multiple, patchy, bilateral, and hyperintense lesions in periventricular areas, the corpus callosum, and deep white matter regions of the fron- tal and parietal lobes. C, D One year after onset, the abnormal MRI signal became slightly more widespread and confluent
Fig. 2 Mutation in the CSF1R gene. DNA sequencing of the patient revealed a heterozygous mutation (c. $2467 \mathrm{G}>\mathrm{A})$ in exon 20 of the CSF1R gene on chromosome 5

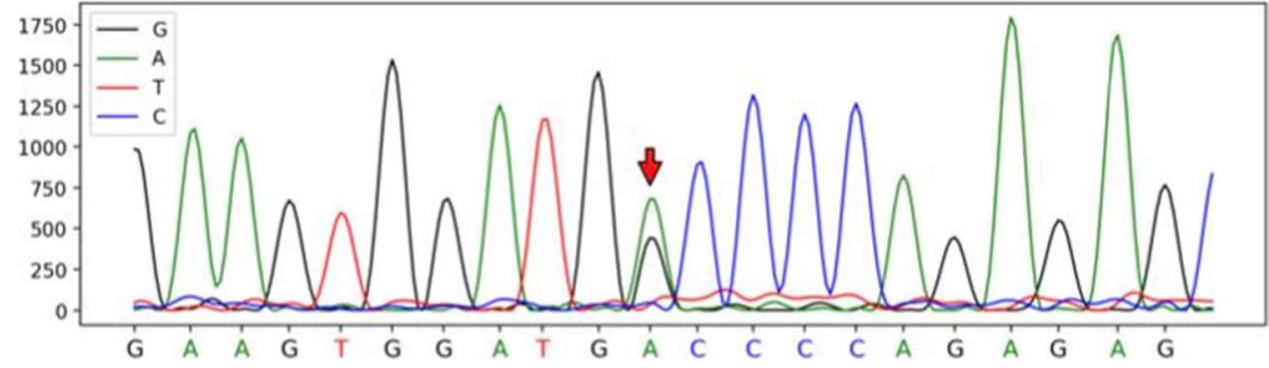

cognitive impairment, and/or psychiatric symptoms. White matter involvement, adult-onset age, and various progressive symptoms make it difficult to differentiate ALSP from NP-SLE when ANA is positive, and no other systems are involved. NP-SLE has been associated with a vast number of heterogeneous syndromes, and to date, there are no laboratory or neuroimaging biomarkers shown to be sufficiently accurate or reliable for use in the clinic [2].

While difficult to elucidate, neuroimaging may provide supportive evidence for ALSP. Bilateral, asymmetrical, or symmetrical, patchy, diffuse, or confluent T2 hyperintensities that are most marked within the periventricular, deep, and subcortical white matter of the frontal lobes are hallmarks of ALSP on MRI. Other early characteristics include signal intensity changes and thinning of the corpus callosum, diffuse cerebral atrophy, and ventricular enlargement [3]. In fact, the first brain MRI of our patient already had the characteristics of ALSP, but there was a misdiagnosis of brain infarction outside of our clinic. The combination of family history, neuroimaging and genetic testing could contribute to avoiding misdiagnosis.

ALSP usually has a rapid course and poor prognosis. It takes 3.9 years from onset to incapacitation and 6.8 years from onset to death [4].Thus, ALSP should be a differential diagnosis for NP-SLE, especially when ANA is positive. Once diagnosed, unnecessary immunotherapy treatment should be avoided. Currently, there is no effective therapy for ALSP.

The variant found in our patient had not been previously reported in the literature. Recently, an increasing number of novel CSF1R mutations have been reported. We guessed that it was also a novel CSF1R mutation. The limitation was that her family segregation was not carried 
out, and without any segregation, characterization of the identified variant was very difficult.

Our case showed that ALSP patients may simultaneously carry positive ANA and encourages clinicians to suspect ALSP when bilateral cerebral white matter and corpus callosum lesions and family history are found.

Availability of data and material The data underlying this article will be shared on reasonable request to the corresponding author.

\section{Declarations}

Conflict of interest The authors declare no competing interests.

Ethical approval Our study complies with the Declaration of Helsinki that the locally appointed ethics committee has approved the research protocol and that informed consent has been obtained from the subject.
Diagnostic criteria for adult-onset leukoencephalopathy with axonal spheroids and pigmented glia due to CSF1R mutation. Eur J Neurol 25(1):142-147. https://doi.org/10.1111/ene.13464

2. Govoni M, Hanly JG (2020) The management of neuropsychiatric lupus in the 21st century: still so many unmet needs? Rheumatology 59(Supplement_5):v52-v62. https://doi.org/10.1093/rheum atology/keaa404

3. Adams SJ, Kirk A, Auer RN (2018) Adult-onset leukoencephalopathy with axonal spheroids and pigmented glia (ALSP): Integrating the literature on hereditary diffuse leukoencephalopathy with spheroids (HDLS) and pigmentary orthochromatic leukodystrophy (POLD). J Clin Neurosci 48:42-49. https://doi.org/10. 1016/j.jocn.2017.10.060

4. Konno T, Yoshida K, Mizuno T, Kawarai T, Tada M, Nozaki H, Ikeda S-I, Nishizawa M, Onodera O, Wszolek ZK, Ikeuchi T (2017) Clinical and genetic characterization of adult-onset leukoencephalopathy with axonal spheroids and pigmented glia associated with CSF1R mutation. Eur J Neurol 24(1):37-45. https:// doi.org/10.1111/ene.13125

Publisher's note Springer Nature remains neutral with regard to jurisdictional claims in published maps and institutional affiliations.

\section{References}

1. Konno T, Yoshida K, Mizuta I, Mizuno T, Kawarai T, Tada M, Nozaki H, Ikeda S-I, Onodera O, Wszolek ZK, Ikeuchi T (2018) 